\title{
De la lógica académica a la lógica civil: una proposición ${ }^{1}$
}

\author{
LUIS VEGA REÑÓN \\ UNED
}

RESUMEN. En el curso de la segunda mitad del siglo XX diversos movimientos, dentro de ámbitos tanto disciplinarios (Filosofía, Derecho) como interdisciplinarios (análisis del discurso, ciencias de la comunicación, humanidades, etc.), han determinado el despegue y desarrollo de los estudios sobre la argumentación. Hoy este campo de estudio se presenta como un nuevo trivium de lógica, dialéctica y retórica, con nuevas posibilidades analíticas, en la línea de una lógica sutil de la pragmática discursiva, y con incitantes retos de constitución teórica o de justificación crítica. Es, en todo caso, la lógica que debería saber una persona educada y, en particular, todo practicante de la Filosofía. Así pues, propongo introducir e implantar en los estudios de Filosofía esta lógica informal o «teoría» de la argumentación, no sólo por la implicación de la propia Filosofía en la práctica de la argumentación o por la necesidad de integrar y dar sentido a los estudios de Lógica y al análisis lógico en ese mismo marco filosófico, sino por la deseable proyección de la «teoría» de la argumentación en la línea de una lógica civil, pendiente de la índole y la calidad de los usos públicos del discurso común - proyección no tan novedosa si se recuerda una tradición
ABSTRACT. During the second half of $20^{\text {th }}$ century, several movements, inside so much disciplinary areas (philosophy, Law) as interdisciplinary ones (discourse analysis, communication, etc.) have determined the rise and development of the studies on argumentation. Today this field of study presents itself as a new trivium of logic, dialectic and rhetoric, with new analytical possibilities in line for a subtle logic of the discursive pragmatics, and with encouraging challenges of theoretical settlement and of critical justification. It is, in any case, the logic that should be known for the cultured people and, especially, for all the students of philosophy. So I propose to implant in the philosophy curriculum this informal logic or «theory» of argumentation, not only due to the implication of the philosophizing in the practice of argumentation, and in order to integrate the studies of Logic and logical analysis in this philosophical frame, but also for a desirable projection of the theory of argumentation in the line of a civil logic, concerned with the shape and improvement of the public discourse -not so novel projection if there is remembered a tradition of our informal logic in Spain been interested in the uses of reason «so much as for the sciences,

${ }^{1}$ Este texto recoge mi intervención en el homenaje a José Luis Escohotado, «Filosofía, ciencia y sociedad en el nuevo siglo», Universidad de La Laguna (18-19 y 25-26 de noviembre de 2004). Incluye algunos datos y resultados del proyecto BFF 2002-03856, financiado por el MCyT. Agradezco a Pablo Ródenas, Carlos Thiebaut y José Francisco Álvarez las facilidades para su publicación en Isegoría. 
guadiana de nuestra propia lógica informal, interesada en los usos de la razón, «así en lo que pertenece a las ciencias, como en lo que toca a los negocios»(P. Simón Abril, 1587). as for the practical affairs»- (P. Simón Abril, 1587).

Para mí es un placer y un honor participar en este homenaje al profesor José Luis Escohotado y agradezco a los organizadores la oportunidad de hacerlo. Conocí al profesor Escohotado en el curso 1969-1970, cuando vine a La Laguna a dar clase de Filosofía en el Instituto de Canarias, entonces Femenino, hoy «Cabrera Pinto», y la facultad de Historia me acogió como PNN de «Filosofía de la Historia». Yo había hecho Filosofía en la Universidad Complutense de Madrid, Filosofía Pura, así que naturalmente al final de la carrera apenas sabía nada. La larga época de profesor en La Laguna, unos ocho años, me sirvió para estudiar de nuevo Filosofía y mi primer mentor en estos estudios fue José Luis Escohotado. Puede que nuestras primeras conversaciones versaran sobre ciertos lugares de Madrid, tal vez lugares de libros - como la trastienda de Fuentetaja - o lugares de sobremesa - como el Gambrinus-, en todos los cuales José Luis, madrileño de Hermosilla, era un reconocido sabio. Puede que se mezclaran ya cuestiones históricas y sociales, o literarias, o didácticas, como las que luego discutiríamos en las veladas que él convocaba en su casa de La Cruz del Señor, donde alrededor de una mesa y al calor de una infusión venía a ser un tipo fuera de lo común, tan comprometido como desengañado, el Jose. Pero, en todo caso, recuerdo que José Luis me impresionó por ser no sólo un sabio, sino un maestro, es decir: un dechado de virtudes intelectuales. Por entonces, a principios de los setenta, la expresión «virtud intelectual», en este país de Congresos Eucarísticos, aún resultaba chocante, si no contradictoria: ningún intelectual podía ser virtuoso. Antes al contrario, el por entonces único inspector de Enseñanza Media de las islas, un cura conocido en los pasillos del Ministerio de Educación como «el virrey de Canarias», me advirtió cuando supo que había bajado de La Laguna a vivir en La Cruz del Señor precisamente: «La vecindad con Escohotado y, peor aún, su trato no son recomendables.»

Pero estábamos hablando de virtudes intelectuales. Una de las virtudes de José Luis que mejor recuerdo es su integridad intelectual —la integridad es un atributo más fuerte que la honradez o que la honestidad, implica entereza de ánimo y una conciencia de la propia dignidad-. A esta virtud acompañaban al menos otras dos de gran valor y peso: la lucidez filosófica y la clarividencia crítica en política y en sociología. $\mathrm{Y}$ todas ellas ejercidas por 
José Luis del modo más natural en el curso de la conversación, discurrían entreveradas de una cuarta prenda de calidad: la sensibilidad cultural, en especial literaria - por lo demás, la vida familiar (la poesía de Julia, los dibujos de Sandra) le ha ido enriqueciendo luego no sólo en ésta, sino en otras sensibilidades estéticas y artísticas-.

Bueno, supongo que el mejor homenaje hacia un hombre de estas virtudes es, amén de reconocérselas, procurar practicarlas. Por mi parte, voy a intentar hacerlo a partir de la primera. Y así, para ser intelectualmente íntegro, he de confesarles que a continuación voy a tratar de venderles una moto: vengo a cantar las excelencias de la lógica civil frente a la lógica académica. En este caso frente no significa contra, aunque aluda a una suerte de contraposición. La lógica académica es la hoy vigente en el área de Lógica y Filosofía de la ciencia, la lógica que en los planes de estudios de Filosofía ejerce de base o de instrumento general del uso de la razón cognitiva, la disciplina que se imparte efectivamente en clase de Lógica: en sustancia, nuestra lógica estándar de primer orden con ciertos complementos técnicos y alguna noticia histórica o filosófica. La lógica civil es la lógica ausente, una lógica que por lo regular no se da entre nosotros, pero - y éste es el puntotambién debería darse. Seré más preciso: lo que debería darse es la Teoría de la argumentación de la que esta lógica se alimenta.

La distinción expresa entre ambas lógicas se remonta, que yo sepa, a Jean Gerson, rector de la Sorbona en el París del primer tercio del siglo XV.

Hay dos lógicas: una, servidora de las ciencias naturales y puramente especulativa, es la que se denomina Lógica casi por antonomasia y es descrita por Pedro Hispano como la que abre la vía de todos los métodos[...]. La otra es la lógica que sirve y presta ayuda principalmente a las ciencias morales, políticas y civiles atendiendo a la inteligencia práctica («De duplici logica», en Una guía de historia de la Lógica, Madrid, UNED, 1997, § 5.0, p. 135).

En España, su manifestación inicial podría ser este significativo título de la Lógica de Pedro Simón Abril (1587): Primera parte de la filosofía, llamada la Lógica o parte racional, la cual enseña cómo ha de usar el hombre del divino don de la razón: así en lo que pertenece a las ciencias, como en lo que toca a los negocios ${ }^{2}$. De ahí parte una tradición digamos guadiana, un tanto sumergida y esporádica, a la que pertenecen Baltasar Gracián en el siglo XVII o Andrés Piquer en el siglo XVIII, hasta alcanzar el siglo XX con la Lógica viva del uruguayo Carlos Vaz Ferreira (1910), a mi juicio la muestra histórica más lúcida de «lógica civil» en español, y llegar incluso a la Nueva filosofía de la interpretación del Derecho de Luis Recaséns Siches (1956),

\footnotetext{
${ }^{2}$ Reeditado en Barcelona, Imprenta Barcelonesa (La Verdadera Ciencia Española, vol. LXVIII), 1886.
} 
que apunta una temprana lógica jurídica de lo razonable ${ }^{3}$ —adelantándose a la publicación de dos clásicos modernos de la teoría de la argumentación y la lógica informal (Perelman \& Olbrechts-Tyteca, 1958, y Toulmin, 1958). Mi denominación: «lógica civil» viene sugerida casi de forma natural por esta tradición, $e . g$. por las alusiones al «trato civil», al discurrir común sobre asuntos públicos o de orden práctico, de la Lógica Moderna de Andrés Piquer (1747) -y de su censura de aprobación por parte de Gregorio Mayàns-. Mayàns asegura, por ejemplo, en la censura citada:

Cualquiera hombre de buen discernimiento recibirá mucha recreación desta Logica escrita principalmente no para gente de Escuela, pues si se destinara para ella no la entenderían los demás, sino para los que profesan la vida activa, i quieren practicar en el trato civil lo mismo que aprenden 4 .

Otros rasgos distintivos de esta lógica civil frente a nuestra actual lógica académica serían los siguientes: $a$ ) sus análisis se refieren a usos del discurso público, en una lengua vernácula, y se atienen a las categorías y las modulaciones pragmáticas del argüir y del argumentar en dicha lengua; por consiguiente, $b$ ) incluyen el reconocimiento de las creencias, actitudes, valores o propósitos, tanto expresos como tácitos, que dan dirección y sentido a los tratos e intercambios argumentativos en marcos discursivos dados; de manera que $c$ ) han de considerar tanto la bondad y la pertinencia argumentativas como la eficacia de la comunicación y la inducción de creencias, decisiones o acciones en el interlocutor o en los destinatarios del mensaje; así que, en definitiva, $d$ ) es una lógica interesada no sólo en unas cuestiones teóricas y analíticas, como la conceptualización, la discriminación o la evaluación de unas razones, pruebas o argumentos, sino en ciertas cuestiones prácticas, $e$. g. en compromisos éticos y consideraciones estratégicas como los de velar por la fluidez de la comunicación intersubjetiva y por la calidad del discurso público. En suma, si ustedes se preguntaran qué es lo que hoy y aquí, en nuestro tiempo y en nuestro medio sociocultural, debe saber de lógica una persona educada, una respuesta sería: por lo menos, lógica civil. Bueno, ¿y dónde se encuentra esa lógica civil? Como ya he sugerido, dentro del campo de la argumentación, por entre algunas de las proyecciones y derivaciones de los estudios sobre la argumentación. Según esto, lo que debería aprender de lógica toda persona educada es, por lo menos, teoría de la argumentación. Ahora bien, es de suponer que todos los filósofos y alevines de filósofos son personas educadas. Luego lo que debería aprender de lógica un filósofo o un alevín de filósofo es por lo menos teoría de la argumentación. Q. E. D.

\footnotetext{
3 Cf. C. Vaz, Lógica viva, 3. ${ }^{a}$ ed., Buenos Aires, Losada, 1945; L. Recaséns, Nueva interpretación de la filosofia del Derecho, 3. ${ }^{\mathrm{a}}$ ed., México, Porrúa, 1980.

4 Véase la edición on line en la Biblioteca virtual Miguel de Cervantes: http://cervantesvirtual.com
} 
Si fuera cierto lo que algunos piensan acerca de los poderes de la mera lógica, ya se habría cumplido mi propósito y se habría terminado esta conferencia. Se trataba de hacerles ver la conveniencia de la teoría de la argumentación y les acabo de dar una prueba deductiva de la necesidad de incorporarla a la formación de la gente educada en general, y de los filósofos en particular. ¿Qué más se puede pedir?

Pero quizás no les haya convencido mi «demostración». Me temo, la verdad, que esa deducción, aun siendo formalmente concluyente al descansar en un argumento lógicamente válido, no convencerá a mucha gente. Así pues, seguramente tendré que aducir mejores razones y habré de recurrir a otros modos de argumentar: por ejemplo, a la dialéctica de las consideraciones plausibles y a la retórica de los motivos convincentes. En suma, me veré obligado a confiar en los recursos del fondo informal de la argumentación $-\mathrm{y}$, si me permiten la observación, he aquí otra prueba de la necesidad de su teoría y de su práctica en medios civilizados como éste y, en general, en cualquier dominio de discurso público-.

Para empezar, puede ser instructivo recordar los motivos de que se emprendiera el camino de la argumentación en la segunda mitad del pasado siglo. $\mathrm{Ni}$ que decir tiene que se trata de un terreno conocido y frecuentado desde antiguo, por lo menos desde que lo abrieron y exploraron hace siglos nuestros clásicos griegos. Pero desde entonces ha llovido bastante y, para los presentes efectos, podemos partir de los años 1950-1960.

En los años cincuenta nuestra lógica formal estándar, la lógica de los conectores veritativo-funcionales y los cuantificadores de primer orden, ya estaba normalizada como disciplina científica e incluso tenía una proyección analítica y metodológica que parecía autorizarla a ejercer como canon o como paradigma del discurso racional. Pero también por entonces aparecen los primeros signos de una inflexión que se irá acusando en la década siguiente. De ahí parten movimientos alternativos en el entorno lógico-filosófico, en el medio escolar y en el ámbito multidisciplinario del análisis lógico. En el entorno lógico-filosófico surgen las vindicaciones del discurso informal $(e . g$., R. Crawshay-Williams, 1957; Ch. Perelman y L. Olbrechts-Tyteca, 1958, y S. Toulmin, 1958) y toma un rumbo pragmático la Filosofía más o menos cómplice, la analítica: el rumbo que señala un Wittgenstein inevitable desde los cincuenta y luego marcan otros como J. Austin, 1962; H. P. Grice, 1967, etc. En los setenta resurge además un interés por las falacias (a partir de Hamblin, 1970) que también confluye en la corriente hacia la pragmática de la argumentación y la lógica informal. Por otro lado, en medios escolares, en los estadounidenses de los años sesenta en particular, concurren varios factores 
-e. g.: radicalización estudiantil, asambleas de campus, insatisfacción producida por la enseñanza reglada de la disciplina de la lógica en los estudios humanísticos - que van determinando la promoción de alternativas informales, como el «Critical thinking» (R. Ennis, 1962); esta alternativa, nacida de una coalición de la lógica informal con las artes del discurso, deviene en los setenta una seria competidora de la lógica establecida en los primeros cursos de Humanidades y de Filosofía, aparte de dominar los Departamentos de Comunicación y Lengua inglesa. No faltarán, en fin, otros movimientos parejos y coetáneos en el ámbito multidisciplinar del análisis lógico que, al igual que las tendencias informales antes indicadas, se vendrán sucediendo y acentuando en el curso de las últimas décadas. Así ocurre en el campo vecino y en parte común de la argumentación jurídica; o en la propia Filosofía (recordemos, $e$. g., la teoría de la acción comunicativa); en retórica y en linguística (e. g., en términos de análisis del discurso); en psicología y en ciencias cognitivas; o incluso en las nuevas fronteras de la lógica con la informática, la inteligencia artificial y las ciencias de la computación [e. g., en casos de razonamiento por defecto (cf. E. Trillas, 1998) y, en fin, la programación de máquinas de argumentación (cf. C. Reed y T. J. Norman (eds.), 2004)] 5 .

En nuestro país, en Filosofía todavía andamos consultando brújulas y desplegando mapas del terreno, como si esos caminos, extramuros de la lógica estándar, estuvieran sembrados de trampas y asechanzas, o pertenecieran a otros viandantes (lingüistas, juristas, etc.). Además, aquí no han tenido una incidencia o una repercusión pareja los motivos o los movimientos mencionados, que actualmente dirigen los cursos de iniciación lógica en Humanidades o están en la base de instituciones y foros académicos en torno a la argumentación en EEUU, Canadá o los Países Bajos. Hoy, no obstante, entre nosotros y en algunos medios, como el jurídico en especial, bien pueden obrar motivos de otro género pero de notable importancia para nuestra lógica civil y, en definitiva, para la suerte de nuestro discurso público. Por ejemplo, a partir de la Constitución de 1978, ha cobrado relieve una motivación mayor o mejor de las sentencias judiciales y el propio Tribunal Constitucional practica y demanda formas de motivación y de justificación altamente exigentes. Por otro lado, en una perspectiva más general, también cabe reconocer no sólo la existencia de ideales regulativos democráticos que propician el recurso a la argumentación, sino otras circunstancias y procesos favorables de hecho, e. g., la pérdida de legitimidad de la autoridad tradicional, o la pérdida de influencia de viejos hábitos doctrinales y de viejas instituciones o corporaciones cerradas, y correlativamente la creciente normalización de la anuencia de los afectados, discursivamente procurada y racionalmente ganada, como nueva fuente de legitimación.

5 Para liberarme de la carga de todas las referencias bibliográficas apuntadas, remito su cita completa a la bibliografía disponible on line en Summa logicae en el siglo xxı, http://logicae. usal.es ( $>$ Estudios de la Lógica $>$ Bibliografía > Argumentación). 
Las razones que acabo de aducir pueden representar motivos genéricos para que un español educado de hoy se interese por la lógica civil, por el estudio de la argumentación y sus usos y aplicaciones, constructivas o críticas, al discurso público sobre asuntos comunes. Apuntarían unos objetivos como los marcados para Filosofía en las sucesivas regulaciones educativas de la enseñanza media desde los noventa (RRDD 1179/1992, 3474/2000, 983/2001 y 832/2002). E irían, por añadidura, en la misma línea que la promoción oficial, gubernativa, de un nuevo talante democrático, límpido y negociador. Pero mi propuesta, al invitarles a tomar el camino de la argumentación, quiere ser algo más concreta y algo menos beatífica: lo que propongo es introducir la teoría de la argumentación en los estudios de Filosofía. Y esta proposición pide razones y motivos específicos.

Antes de pasar a este terreno de la motivación y la justificación vayan por delante unas precisiones sobre la propuesta misma. No pretendo sustituir la lógica establecida por otro tipo de estudios y análisis del discurso, sino complementar esa lógica formalizada, sistemática y exacta, con una lógica más sutil y contextualizada, más atenta a la pragmática de nuestras acciones e interacciones discursivas. Por otro lado, aunque la lógica sea hoy una disciplina no sólo académica, sino ocupacional y tecnológica, con mayor proyección en las escuelas de la Politécnica que en las facultades clásicas (Filosofía, Matemáticas), aquí me limitaré a considerar este marco académico tradicional, en particular el caso de Filosofía. Sólo me referiré al lugar y a la función del estudio de la lógica en los estudios de Filosofía. Creo además que, a través de la ampliación o complementación de la lógica estándar con la teoría de la argumentación y en una línea de atención a los usos reales del discurso, la propia disciplina podría recuperar el sentido y la significación que ha venido perdiendo en las últimas décadas en los estudios de filosofía. Así que mi propuesta tiene un doble filo: de un lado, sostengo que la teoría de argumentación es una parte sustancial de la lógica que deberían saber los filósofos y, por lo tanto, deberían aprender los estudiantes de Filosofía; de otro lado, afirmo que este conocimiento de la teoría de la argumentación puede dar sentido, contenido y uso a lo que ya se haya aprendido de lógica estándar en Filosofía.

Las razones que obran en favor de la primera tesis descansan en las relaciones que ligan, a mi juicio de modo sustancial, el oficio y el ejercicio de la Filosofía a la práctica de la argumentación. Las razones que obran en favor de la segunda tesis son de orden histórico y sociológico: tienen que ver con la situación institucional de la lógica en España. Unas y otras también pueden servirnos para recordar las dos virtudes intelectuales de José Luis Escohotado que había mencionado antes: su lucidez filosófica y su clarividencia crítica. Cuando menos intentaré seguir su ejemplo al ocuparme de esas razones empezando por las relativas a la situación institucional y académica de la lógica. 
III

La consideración de la situación institucional y académica de la lógica envuelve aspectos muy dispares: desde supuestos históricos hasta tendencias de la investigación en curso, pasando por disposiciones administrativas, condiciones y medios socioculturales - círculos de más o menos establecidos de influencia, la Sociedad de Lógica, Metodología y Filosofía de la Ciencia en España (SLMFCE) y sus congresos cuatrienales, revistas como Theoria o Teorema, etc.-, y determinantes académicos de diverso orden - planes de estudios, textos y manuales disponibles, etc.- . Pero ahora sólo podré ser alusivo y esquemático: a los interesados en fuentes y datos estadísticos, y en las demás referencias de detalle, les remito a mi largo informe on line, «La lógica del siglo XX en España», véase Summa logicae en el siglo XXI, http://logicae.usal.es ( Estudios de la Lógica > Historia de la Lógica).

Según es bien sabido, la lógica conoció entre finales del siglo XIX y principios del XX una profunda transformación tanto en su constitución como en su cultivo. Este cambio no tuvo mayor repercusión en España hasta los años sesenta y, aun entonces, parecía un empeño con más pretensiones que conocimientos técnicos. Se trataba de sustituir una lógica no sólo rancia, sino vinculada a la filosofía neo-escolástica por otra no sólo moderna sino científica, por la «lógica matemática». Pero eran voces de filósofos las que pedían la sustitución y fue en medios filosóficos - con la complicidad de otras recepciones coetáneas como la de la filosofía analítica-donde antes se hizo efectiva la recepción de la nueva lógica. Tras un proceso de implantación y expansión académica en los sesenta y setenta, llegó su normalización institucional con el establecimiento del área de Lógica y Filosofía de la Ciencia (RD 1888/1984), que contribuyó a determinar una creciente autonomía y profesionalidad del cultivo de la lógica en las décadas ochenta y noventa; la tendencia a la especialización profesional se ha visto luego reforzada por la política seguida en investigación (evaluación de sexenios, proyectos, etc.). Los ochenta también trajeron la normalización académica con la nueva organización de planes de estudios que distribuía las materias en troncales, obligatorias y optativas (RD 1497/1987; para Filosofía, RD 1467/1990). La Lógica viene a ser troncal, parte del núcleo común de la formación filosófica, bajo esta curiosa descripción: «Teoría del razonamiento y la argumentación correcta, rudimentos de metalógica, filosofía de la lógica» ${ }^{6}$. Aparte de esta posición central de la disciplina, los temas y las cuestiones lógicas

\footnotetext{
6 Esa presunta teoría del razonamiento y de la argumentación correcta no existe -al menos, que se sepa-; desde luego, no consiste en algún sistema al uso de lógica de primer orden. La expresión «rudimentos de metalógica» suena un poco chocante, algo ruda para el caso. Y la fillosofía de la lógica suele ser una materia optativa, si algo es, en los planes de estudios efectivamente cursados. Así pues, convengamos en que el (la) o los (las) responsables de esta descripción no estaban, al redactarla, en su mejor momento.
} 
pasan a ocupar varios y diversos lugares de visita, con carácter obligatorio $\mathrm{u}$ opcional, en los currículos de Filosofía. Según mis datos, relativos a las ofertas de Filosofía en las 23 universidades en que se cursa - cinco de ellas privadas-, el ámbito temático de la lógica contaba con 12 materias obligatorias y 59 optativas en el curso 2003-2004. No es nada desdeñable una oferta de 71 asignaturas en los planes de estudios del país, aparte de la Lógica troncal y omnipresente. Esta presencia académica se ve corroborada por otros indicadores dentro del área de Lógica y Filosofía de la Ciencia, donde le corresponde un 35 por 100 de la oferta global del área en el $2 .^{\circ}$ ciclo. En el $33^{\text {er }}$ ciclo los números bajan y la proporción desciende: para el curso 2003-2004, sólo nueve universidades ofrecían cursos o líneas de trabajo relacionados con temas de lógica en sus programas de doctorado y únicamente había dos programas específicos de Lógica. Pero la verdad es que no faltan indicadores curriculares universitarios mucho menos alentadores. Baste recordar los estudios de Humanidades, que también incluyen algunas materias de Filosofía: de los 19 planes que he revisado, resulta una oferta reducida a tres asignaturas de Lógica. Son otras materias del área las más agraciadas o atractivas en Humanidades; entre ellas sobresalen los estudios de Ciencia, Tecnología y Sociedad - la industria escolar más floreciente en las dos últimas décadas -, seguidos a distancia por la Filosofía del lenguaje.

Ahora bien, cuando la moral cae por los suelos es al considerar la suerte de la lógica en Filosofía del Bachillerato, como consecuencia de una serie de reducciones acumuladas: una reducción de los años del Bachillerato, dentro de ella una reducción de los estudios de Filosofía y, dentro de esta última, una reducción y trivialización de las unidades didácticas de Lógica hasta su desaparición de la práctica escolar en muchos casos. Me limitaré a mencionar la evolución sintomática de las ideas del famoso Gustavo Bueno: en su manual de Filosofía de 1955, dedicaba a la lógica 11 lecciones (39,2 por 100 del programa) y $144 \mathrm{pp}$. (42,2 por 100 del texto); en el número 23 , enero de 2004, de Catoblepas, el planfleto online de www.filosofia.org, presenta un programa de Filosofía, Symploké, en el que la lógica se ha desvanecido o derruido para alzar en su lugar una gnoseología.

Por lo demás, la consideración de otros aspectos relacionados con la productividad científica, como las publicaciones en revistas o las contribuciones a congresos, podría llevar a conclusiones parecidas: la lógica parece mantener un prestigio y una relevancia que contrastan con su escasa o nula repercusión sobre el pensamiento o el ensayo filosófico que hoy se practica y publica en España. Más aún, la situación de la lógica en los estudios de Filosofía revela desajustes y desequilibrios paralelos en el propio medio académico. Dos, al menos, serán especialmente familiares para todo el que frecuente una facultad de Filosofía: 1) la desproporción existente entre la oferta académica y la demanda real, en vista del escaso interés que los estudios lógicos suscitan entre los estudiantes de Filosofía en general - por no hablar 
de la indiferencia que suelen mostrar hacia el análisis lógico los demás colegas de la facultad-, desinterés que hoy incluso ha prendido y se extiende dentro del área de Lógica y Filosofía de la Ciencia ${ }^{7}$; 2) la distancia que, de acuerdo con esta y otras circunstancias, media entre la presencia oficial de las materias de Lógica en los planes universitarios de Filosofía y su significación filosófica o su incidencia real en los estudios filosóficos mismos.

De ahí se desprende una rara sensación que compartimos muchos, aunque no tantos estemos dispuestos a confesar: la sensación de que la normalización de la lógica en los estudios de Filosofía se ha producido en falso, por no decir in vacuo, en el vacío. Esta sensación viene inducida por la impresión de habérselas con un cuerpo extraño: es la propia lógica la que parece extraña a la mayoría de los profesores y estudiantes de Filosofía. Hay varios motivos y señales de este síndrome de - pongámonos tiernos - «desencuentro». Un motivo es la existencia misma de una lógica matemática y ajena a la Filosofía, como la desarrollada a partir de ciertos legados técnicos del siglo Xx: modelos, estructuras algebraicas, recursividad, teoría de la computabilidad, programación lógica. «Por lo tanto, ¿no será mejor ubicar la Lógica en Matemáticas?», se preguntan retóricamente algunos lógicos que quizás se sorprenderían de lo que piensan al respecto algunos matemáticos, no muy dados a considerar de la familia todo lo que circula por ahí bajo el nombre de «Lógica»y, en particular, de «análisis lógico»-. Hoy, en todo caso, la asociación más popular liga la lógica con la Informática y sólo los más viejos del lugar parecen recordar su liaison en los años sesenta-setenta con la Filosofía. En cuanto a los síntomas, recordemos la esquizoide experiencia del profesor de Lógica que vive dividido entre los resultados de una investigación casi autista, que sólo puede confiar a un círculo de iniciados, y las rutinas escolares que ha de desplegar y difundir como conocimiento público - materia troncal - entre sus despegados alumnos y ante la indiferencia de sus colegas. Pero el desinterés por la lógica no sólo es notorio entre los principiantes de $1{ }^{\text {er }}$ curso. También se extiende entre los sesudos doctorandos de 3 er $^{\text {er }}$ clo, sesgo que se da tanto en Filosofía como en otras facultades clásicas, concretamente en Matemáticas. Puede apreciarse esta tendencia en los dos cuadros siguientes, que he elaborado a partir de las tesis de doctorado recogidas en la base de datos Teseo desde sus inicios, 1976-

\footnotetext{
7 Resulta significativa la evolución de los porcentajes de contribuciones en los cuatro Congresos de la SLMFCE, celebrados hasta el momento, y de contenidos en revistas características del área como Theoria y Teorema. Para datos al respecto, me remito una vez más a mi informe on line, «La lógica del siglo XX en España», en el portal Summa logicae en el siglo XXI, http://logicae.usal.es (> Estudios de la Lógica > Historia de la lógica). Coincide además con el creciente interés de los lógicos españoles por publicar en revistas especializadas y acreditadas internacionalmente pero muy alejadas del -o inaccesibles al- común de los lectores de Filosofía en España, de modo que los procesos de profesionalización y autismo se suponen convergentes.
} 
1977, hasta 2001-2002, las registradas en mi última consulta de enero de 2004, bajo los descriptores «lógica» y afines - de casi 400 registradas, entre las que se incluyen títulos que responden a muy dispares usos de esos términos, e. g. «lógica de las relaciones de parentesco», «lógica de la conducción vial», «lógica de los sentimientos», etc., han resultado pertinentes 289 tesis en el presente contexto-. El cuadro I se refiere al centro de lectura de las tesis; el cuadro II, al área de conocimiento o temática correspondiente según cabe inferir de los descriptores y de los resúmenes. Creo que ambas referencias son parámetros significativos de las tendencias de investigación en curso. Por lo demás sólo contabilizo, en ambos casos, las más relevantes en el presente contexto.

Cuadro I

\begin{tabular}{crrrrr}
\hline \multirow{2}{*}{ Periodo } & \multicolumn{3}{c}{ Centro en el que se han leido las tesis ${ }^{8}$} & \multicolumn{1}{c}{ Totales } \\
\cline { 2 - 6 } & Fac. Filosofía & FF. Ciencias & ETS Politécnica & Parcial/Referencia \\
\hline $76 / 77-79 / 80$ & $9[40,9 \%]$ & $8[36,3 \%]$ & $1[4,5 \%]$ & 18 & 22 \\
$80 / 81-89 / 90$ & $46[58,4 \%]$ & $18[18,9 \%]$ & $16[16,8 \%]$ & 80 & 95 \\
$90 / 91-99 / 00$ & $48[32,4 \%]$ & $32[21,6 \%]$ & $59[39,5 \%]$ & 139 & 149 \\
$00 / 01-01 / 02$ & $5[21,7 \%]$ & $1[4,3 \%]$ & $16[69,5 \%]$ & 22 & 23 \\
[sumas: & 108 & 59 & 92 & 259 & $289]$ \\
\hline
\end{tabular}

\section{Cuadro II}

\begin{tabular}{cccccc}
\hline \multirow{2}{*}{ Periodo } & \multicolumn{3}{c}{ Áreas y campos temáticos } & \multicolumn{2}{c}{ Totales } \\
\cline { 2 - 6 } & "Lógica-F. ${ }^{a} C^{a{ }}$ & Matemáticas & Comp./IA/Inform. & Parcial/Referencia \\
\hline $76 / 77-79 / 80$ & $16[72,7 \%]$ & $3[13,6 \%]$ & $1[4,5 \%]$ & 20 & 22 \\
$80 / 81-89 / 90$ & $60[63,1 \%]$ & $13[13,6 \%]$ & $12[12,6 \%]$ & 85 & 95 \\
$90 / 91-99 / 00$ & $61[41,2 \%]$ & $11[7,3 \%]$ & $67[44,9 \%]$ & 139 & 149 \\
$00 / 01-01 / 02$ & $8[34,7 \%]$ & $2[8,6 \%]$ & $12[52,1 \%]$ & 22 & 23 \\
[sumas: & 145 & 29 & 92 & 266 & $289]$ \\
\hline
\end{tabular}

Estos datos, a los que cabría añadir otras indicaciones en el mismo sentido de una recesión progresiva de la lógica dentro de su propia área ${ }^{10}$, pue-

\footnotetext{
${ }^{8}$ Hay centros no incluidos aunque cuenten con lecturas de tesis: facultad de Filología ( 9 tesis leídas), Derecho (5), CC. Económicas (2), Medicina (2), Psicología (4), Ciencias de la Información (3). En la Politécnica, la ETS más frecuentada es Informática (70), seguida de Telecomunicaciones (12) e Industriales (10).

${ }^{9}$ Tampoco están incluidos otros campos temáticos, como Derecho (8), Ciencias Cognitivas (17), etc.

${ }_{10} \mathrm{Cf}$. los informes sobre otros indicadores académicos e institucionales que Enrique Alonso ha publicado en la red www.elvira.lllf.uam.es/ logicaww.htm. Repárese en que los datos correspondientes en ambos cuadros tanto al primer periodo, 1976-1980, como al último, 2001-2002, sólo tienen un carácter indicativo muy parcial, sobre todo el último, pues distan mucho de cubrir décadas completas
} 
den producir la impresión de que el cultivo de la lógica en Filosofía genera, si acaso, más autosuficiencia que provecho. Y la impresión se refuerza cuando se observa que el relativo pero creciente aislamiento de la lógica dentro de «su» facultad - e incluso de su área - viene acompañado de tendencias internas hacia la especialización ensimismada del análisis lógico. No digo que esto sea malo de suyo. Pero me temo que no es la mejor estrategia para dar significado y sentido al cultivo de la Lógica dentro de los estudios de Filosofía. En cambio, sería más aconsejable contextualizar y orientar los servicios de la lógica en la línea del análisis del discurso filosófico y, por ende, en el marco general de la teoría de la argumentación. Repito que no se trata de excluir o de sustituir la lógica estándar y, menos aún, el análisis lógico formal, sino de reacomodar sus servicios disciplinarios y hacerlos fructificar en el marco al que, según nuestros planes de estudios de Filosofía, se supone que corresponden; me refiero, en especial, al marco de la teoría y la práctica del discurso filosófico.

Pero hay razones más poderosas que unos meros motivos de oportunidad para incorporar la teoría de la argumentación a los estudios de Filosofía y, en concreto, para relacionar dentro de este marco la lógica académica con la lógica civil. Consideremos de nuevo la cuestión anterior: ¿Qué debería saber de lógica un filósofo?

La lógica, claro está, puede concebirse y practicarse de muy diversas maneras en Filosofía. Su cultivo, para empezar, admite:

a) Una concepción más bien sistemática que considera la lógica como materia teórica o analítica autónoma, donde cuentan sobre todo los desarrollos semánticos formales, o los que proceden en términos de secuentes y de teoría de la prueba, o los relacionados con la teoría de la computación, etc.

b) Pero también es perfectamente admisible, al menos en teoría, una concepción más filosófica y pendiente de los conceptos lógicos básicos - consecuencia, necesidad, identidad, etc.—, o de los supuestos ónticos y epistémicos de los sistemas lógicos disponibles.

c) Así como desde luego cabe, sobre todo en la práctica, adoptar una concepción instrumental del análisis lógico, sea c.l) orientada al análisis de las estructuras teóricas y los métodos de la inferencia científica, sea $c .2$ ) centrada en el análisis de la inferencia o del discurso común y abocada al estudio de los argumentos y las argumentaciones reales y efectivas.

No son alternativas únicas, ni son excluyentes entre sí; antes bien, podrían beneficiarse mutuamente aunque las variantes no dejaran de poner más énfasis o mayor acento en una de las opciones frente a otras. En fin, todas ellas 
podrían convenirle a un filósofo, incluso las más teóricas o más técnicas en la línea $a$ ), pues cabe suponer que los filósofos también están llamados a hacer de modo competente filosofía de la lógica.

En cualquier caso, lo cierto es que a los estudiantes de filosofía, en razón de la propia índole discursiva de los textos y los saberes filosóficos, les vendría muy bien tener conocimientos de teoría de la argumentación (o de lo que hoy se entiende bajo este epígrafe y es un «entre-saber-y-arte» que se busca). Dicha «teoría» versa sobre dominios y marcos de construcción y confrontación de alegatos, razones y estrategias de prueba y contra-prueba, que revisten especial interés para los estudiosos y los practicantes de la Filosofía debido a las proposiciones y las argumentaciones filosóficas mismas. Veamos por qué ${ }^{11}$.

En Filosofía, ya se sabe, ninguna tesis será tan incontestable que no admita contra-argumentación alguna, ni tan absurda que excluya cualquier intento de rehabilitación razonable. Así pues, no son cuestiones netas de validez o invalidez formal las que aquí se ventilan en principio, sino cuestiones graduales y comparativas como una mayor o menor plausibilidad o justificación o un poder de convicción relativo, a la luz de lo que se aduce o se podría aducir en contra de la proposición avanzada o en favor de la postura opuesta. Lo que más importa en tales casos no es disponer de un lenguaje o un sistema lógico —ni de un ábaco de decisión como el que soñara Leibniz ${ }^{12}$-, sino contar con las perspectivas e instrumentos analíticos, contextuales e informales de una teoría de la argumentación. Según esto, un filósofo debería conocer no sólo la lógica sistemática de la convalidación o la invalidación formal, sino la pragmática de la conversación y la lógica sutil de las bazas, los triunfos y las reglas del juego de dar y pedir razones, persuadir o disuadir a alguien de algo. La teoría de la argumentación viene a ser, en suma, una buena compañía para la Lógica troncal, la lógica que anida en el tronco mismo del árbol de la Filosofía.

Pero, además, hay razones para pensar que ningún servicio de una Lógica troncal a la Filosofía se diría más pertinente que el estudio y análisis de la argumentación, de los argumentos filosóficos en particular. Como todo el mundo sabe, no es fácil explicar en qué consiste la Filosofía o qué hay que hacer para hacer filosofía. Lo cierto cuando menos, creo, es que hay que argüir y argumentar y que la Filosofía se distingue, entre otras cosas, por el

${ }^{11}$ No pretendo dar explicaciones exhaustivas sino, simplemente, hacer caer en la cuenta de un par de razones obvias. Pueden verse otros motivos y consideraciones en J. Passmore, «The place of argument in philosophy», Symposium sobre la argumentación filosófica, México, UNAM, 1963, pp. 23-36, o en el más comprensivo N. Rescher, Philosophical reasoning. A study in the methodology of philosophizing, Oxford, Blackwell, 2001.

12 «... Cuando surja una controversia, no habrá ya necesidad de discusión entre dos filósofos que entre dos calculadores. Bastará, en efecto, con tomar la pluma en la mano, sentarse ante el ábaco y decirse uno a otro: Calculemos» (cf. I. M. Bocheński, Historia de la lógica formal, Madrid, Gredos, 1966, \& 38.09, p. 290). 
uso de unos tipos más o menos característicos de argumentos y contra-argumentos (argumentos fundacionales, trascendentales, analíticos; reducciones al absurdo, regresiones o progresiones al infinito, etc.). Más aún: la práctica de la argumentación es consustancial a la práctica de la filosofía. Esta vinculación no sólo implica que la aceptación o el rechazo de una proposición filosófica deben descansar en la confrontación de argumentos. Implica, más radicalmente, que el propio significado de tal proposición depende de las razones aducidas: no podemos saber qué dice precisamente o qué quiere decir una tesis filosófica antes o al margen de sus pruebas y contrapruebas. Así que la interpretación en filosofía no es sólo una cuestión de hermenéutica, ni de formalización precisa; también requiere las luces de la pragmática argumentativa. No puedo extenderme en este punto: sólo recordaré que si, en la línea cognitiva de R. Brandom, hay un juego de compromisos y de habilitaciones inferenciales que determinan y precisan los conceptos empleados, un juego similar determina y precisa el significado de las proposiciones filosóficas que se sostienen. Sea $\mathrm{P}$ una tesis filosófica. Sé lo que quiero decir al decir $\mathrm{P}$ y los demás pueden entender lo que digo, cuando está claro, por un lado, a qué me compromete la aserción de P, por ejemplo: qué objeciones deberé arrostrar y de qué pruebas dispongo en favor de $\mathrm{P}$ o contra las objeciones mismas; y cuando también queda claro, por otro lado, qué estoy autorizado a derivar de P, por ejemplo: qué deberá aceptarme quien asuma, siquiera provisionalmente, esta posición y qué contra-pruebas debería aducir a su vez si no la asumiera. De lo cual se desprende que un aforismo o una sentencia puede contener tal vez un pensamiento profundo, una revelación trascendental o una verdad última o primera, pero mientras no se argumente será difícil conocer tanto su contenido como su calidad filosófica. Los hacedores de frases no son gente de fiar en Filosofía.

Por lo demás es evidente que los argumentos y la argumentación también revisten suma importancia para la lógica misma. Constituyen, sin ir más lejos, la vía más familiar de acceso a los usos de la implicación, las ideas de ilación y las relaciones de consecuencia. Y, de hecho, las referencias al análisis formal de la argumentación o al estudio de argumentos for malmente válidos recorren nuestros manuales, desde los clásicos (e. g., Sacristán, 1964; Mosterín, 1970; Garrido, 1973, y Deaño, 1974) hasta los m ás modernos (e. g., Badesa, Jané y Jansana, 1998; Falguera y Martínez Vi lal, 1999; Manzano y Huertas, 2004) ${ }^{13}$. Más aún, el partir de la argument ción para llegar a la formalización lógica cuenta incluso con el beneplácito iternacional: es una estrategia didáctica bendecida por el Commitee on Jogic and Education de la Association of Symbolic Logic (ASL) ${ }^{14}$.

\footnotetext{
13 Hasta tal punto que sólo sé de un manual que ignore esta asociación y hable i icamente de «argumento» en contextos del tenor: «función de $n$ argumentos» [Zalabardo (2000), z 02].

14 «Guilelines for Logic education», The Bulletin of Symbolic Logic, vol. I, : im. 1, 1995, pp. 4-7.
} 
En suma, si la teoría de la argumentación conviene tanto a lógicos como a filósofos, ¿por qué no integrarla formalmente en los estudios de Filosofía? El único reparo que cabría a estas alturas sería el de no saber muy bien a qué atenernos ante esta propuesta, no saber muy bien qué significa o a qué nos compromete. Para reducir o desvanecer esta última línea de resistencia, veamos el contenido de la propuesta.

Llegados a este punto y a esta hora, seré telegráfico para no abusar de su paciencia. Así que mi propuesta de una teoría de la argumentación se va a concretar en dos puntos básicos: una idea de lo que es argumentar y una visión del campo de la argumentación que deje traslucir sus perspectivas o dimensiones y sus problemas.

Creo que argumentar es, en general, conversar. Más específicamente consiste en una manera de dar cuenta y razón de algo a alguien o ante alguien en el curso de un debate, siquiera posible. Envuelve procesos de comunicación e interacción entre interlocutores o entre un «locutor» y un auditorio en marcos más o menos institucionalizados de discurso: la interacción puede ser, a su vez, viva y en directo, o estar congelada y darse en diferido, e. g., a través de un texto -incluidos ciertos casos intermedios como un chat entre internautas -. Por otro lado, toda argumentación dada es un iceberg, donde la parte expresa asoma sobre una parte mayor, de fondo y tácita, así que la consideración cabal del argumento, i. e., tanto su entendimiento, como su análisis o evaluación, también suponen una interpretación, una explicitación de lo implícito en su contexto y en su marco discursivo. Importa tener en cuenta esta complicidad entre la argumentación y la interpretación especialmente cuando se trata de entender y juzgar las proposiciones filosóficas: sin sus trasfondos argumentativos no llegaríamos a entender cabalmente qué significan, pero sólo a través de explicitaciones o interpretaciones podremos determinar esos trasfondos precisos.

En consonancia con esto no veo el campo de la argumentación como un terreno distribuido en compartimentos estancos: demostraciones concluyentes, pruebas más o menos sólidas, alegatos convincentes, falacias. Más bien me lo imagino como un campo común en el que pueden medrar tanto las buenas como las malas hierbas y, por ejemplo, pueden darse entre ellas variantes de la argumentación falaz que se extienden desde el extremo del error ingenuo, inconsciente o no intencionado (paralogismo), hasta el extremo del engaño urdido con malas artes, subrepticia y deliberadamente (sofisma), de modo que si bien esta extensión forma una especie de «continuo», no se borra la distinción y separación entre ambos extremos, al igual que una gama de grises no difumina la diferencia entre el polo más blanco y el 
más negro. Lo mismo cabe pensar de la distinción entre los buenos y malos argumentos, una distinción no sólo pragmática sino normativa y por añadidura sujeta a diversos criterios según la perspectiva o la dimensión argumentativa que se considere.

La tradición de los estudios en torno a la argumentación ha venido destacando tres perspectivas que se suponen correspondientes a tres aspectos o consideraciones básicas del discurso argumentativo mismo: el argumento como producto, el argüir como procedimiento y la argumentación como proceso. Son tres las perspectivas que en honor del padre de estos estudios, Aristóteles, suelen denominarse lógica (o analítica), dialéctica y retórica ${ }^{15}$. Valga el siguiente esquema:

\begin{tabular}{lll}
\multicolumn{1}{c}{ Perspectiva } & \multicolumn{1}{c}{ Aspecto de la argumentación más resaltado } \\
\hline LÓGICA & $\Rightarrow \quad \begin{array}{l}\text { Productos } \approx \text { argumentos textuales, cuya forma básica se compone } \\
\text { de un conjunto de premisas, un nexo inferencial y una conclusión, }\end{array}$ \\
DIALÉCTICA & $\Rightarrow \quad \begin{array}{l}\text { Procedimientos } \approx \text { fases y variantes + reglas y convenciones de la } \\
\text { interacción argumentativa, codificadas en una normativa del deba- }\end{array}$ \\
RETÓRICA & $\Rightarrow \quad \begin{array}{l}\text { Procesos } \approx \text { entros partes o dos papeles, proponente y oponente. } \\
\text { personas, audiencias o auditorios, con el fin de que adopten ciertas } \\
\text { creencias, decisiones o acciones. }\end{array}$
\end{tabular}

No faltan otras perspectivas menos generales o menos relevantes. Pero la raigambre y la importancia de estas tres han propiciado la conversión de los aspectos atendidos o destacados por cada una de ellas en dimensiones constitutivas de la argumentación misma. Por lo menos, pueden servirnos para esbozar a su luz una caracterización de los tres puntos de vista en razón de sus respectivos focos de atención, paradigmas argumentativos y objetivos analíticos. Valga la caracterización siguiente:

\begin{tabular}{|c|c|c|c|}
\hline & Foco central & Paradigma & Objetivo: determinación de \\
\hline L. & $\begin{array}{l}\text { Argumentos (pro- } \\
\text { ductos) }\end{array}$ & $\begin{array}{l}\text { Prueba racional - de- } \\
\text { mostración- }\end{array}$ & $\begin{array}{l}\text { Validez o corrección según criterios semán- } \\
\text { ticos lógicos o metodológicos. }\end{array}$ \\
\hline D. & $\begin{array}{l}\text { Procedimientos de } \\
\text { interacción y de } \\
\text { confrontación en- } \\
\text { tre roles argumen- } \\
\text { tativos }\end{array}$ & Debate razonable & $\begin{array}{l}\text { Actuaciones buenas/malas (falaces) median- } \\
\text { te análisis pragmáticos y contextuales del } \\
\text { proceder según reglas. }\end{array}$ \\
\hline R. & $\begin{array}{l}\text { Procesos de comu- } \\
\text { nicación e influjo } \\
\text { interpersonal }\end{array}$ & Discurso persuasivo & $\begin{array}{l}\text { Recursos y estrategias eficaces para inducir } \\
\text { creencias, disposiciones o acciones. }\end{array}$ \\
\hline
\end{tabular}

15 Su implantación actual procede de un influyente artículo de Wenzel (1980) y de asunciones posteriores, e. g. la de Habermas (1983); cf. su revisión: J. Wenzel, «Three perspectives on argu- 
Este esquema tiene la virtud añadida de sugerir dos problemas teóricos principales, a mi juicio, en el momento actual de los estudios sobre la argumentación. Son un desafío de orden general y una cuestión interna, a saber:

1. El desafío estriba en cómo pasar desde los puntos de vista o perspectivas disponibles hasta una visión integrada del campo de la argumentación. Es decir: ¿cabe esperar una teoría general y comprensiva de la argumentación? No parece que las tendencias finiseculares hacia la sectorialización del análisis - tan notorias, por ejemplo, en el tratamiento monográfico de las falacias-, así como las tensiones multidisciplinarias de los estudios sobre el discurso argumentativo, favorezcan tales expectativas, aunque tampoco faltan los ensayos de unificación e incluso de integración transdisciplinaria. En todo caso, mientras no se disponga de una visión comprensiva e integrada, de esa suerte de «saber-y-arte» que se busca, convendrá referirse al «campo» antes que a la «teoría» de la argumentación.

2. El problema interno reside en la determinación de las relaciones entre la validez o bondad y la eficacia argumentativas, puesto que no son infrecuentes los argumentos malos o falaces pero convincentes, así como los argumentos válidos o correctos pero carentes de éxito; en suma, la inducción retórica no es el correlato lógico o automático de la corrección metodológica o de la bondad dialéctica del argumento, como, en la acción lingüística en general, el efecto perlocutivo pretendido no se sigue de la fuerza ilocutiva empleada. Se trata de una cuestión bifronte o con una doble dimensión, filosófica y empírica. De una parte cuenta el punto filosófico de la justificación: por qué argumentar bien, en vez de mal, si este mal proceder resulta precisamente el más eficaz o el que obtiene más éxito o, cuando menos, un buen proceder conforme a las reglas o criterios puede no constituir por sí mismo una garantía de éxito, de convencimiento ${ }^{16}$. De otra parte cuenta la investigación empírica de la frecuencia relativa de los casos de no correlación o desproporción entre calidad y eficacia. Pues bien, por más que sorprenda a los desengañados o suspicaces, los estudios experimentales en curso vienen mostrando que, de hecho, prevalece la correspondencia entre el mayor cuidado y respeto de la calidad argumentativa del discurso y su mayor eficacia, sobre su presunta divergencia o inarmonía práctica ${ }^{17}$.

Otras cuestiones que parece obligado mencionar en este contexto por su relevancia crítica y filosófica son las proyecciones éticas y estratégicas del

ment», en R. Trapp y J. Schuetz (eds.), Perspectives on argumentation, Prospect Highs (IL), Waveland Press, 1990, pp. 9-26.

16 En la cuarta y última parte de mi Si de argumentar se trata (Barcelona, Montesinos, 2003) puede verse un planteamiento de esta cuestión que, a mi juicio, es sustancial y dista de estar resuelta.

17 Cf. Daniel J. O'Keefe, «The potencial conflict between normatively-good argumentative practice and persuasive success: evidence from persuasion effects research», en F. H. van Eemeren et al. (eds.), Anyone who has a view. Theoretical contributions to the study of argumentation, Dordrecht, Kluwer, 2003, pp. 309-318. 
estudio de la argumentación en la línea de una posible y deseable lógica civil. Estas proyecciones tienen que ver con la asunción y la depuración de responsabilidades respecto del discurso público. Como ya no me podré detener en la discusión de este punto, me limitaré a apuntar dos muestras de lo que cabría plantear y discutir en tal sentido:

1) La primera es un caso de, digamos, lógica civil «privada», como el suscitado por la suspicaz estrategia de Schopenhauer a la hora de tener la razón, esto es: hacerla valer, mediante un buen número de ardides y estratagemas, frente a cualquier contrario ${ }^{18}$.

En palabras de Schopenhauer: «Maquiavelo escribió al príncipe que aprovechase cada instante de debilidad de su vecino para atacarle, porque de lo contrario aquél se aprovecharía a su vez de los suyos. Si dominasen la fidelidad y la franqueza, sería muy distinto; pero como su uso no es frecuente, también está permitido dejar de utilizarlas, o de lo contrario uno se verá mal pagado. Lo mismo ocurre en la discusión. Si doy la razón al adversario mientras parezca tenerla, será difícil que él haga lo propio en el caso inverso. Más bien acudirá a medios ilícitos. Por tanto, yo debo hacerlo también. Es fácil decir que debe buscarse únicamente la verdad, sin prejuicios en favor de la propia tesis. Pero no hay que suponer que el otro también lo haga. Ésta el causa por la que nosotros también tenemos que abstenernos de pretenderlo» (cf. edic. 1967, p. 47, nota 3; edic. 1996, pp. 74-75, nota 3). En suma, lo que se recomienda por buenos motivos al parecer - entre otros, la fragilidad y vanidad de la naturaleza humana - es engañar para no ser engañado, recomendación más paradójica e inviable de lo que parece a primera vista. Prueben a adoptarla como estrategia de comunicación e interacción discursiva o, peor aún, a generalizarla.

2) La segunda muestra es un caso de, digamos, lógica civil «pública». Se trata del tema de concurso propuesto por la Real Academia de Ciencias y Letras de Berlín en 1778, a instancias de Federico II y por sugerencia de d'Alembert: «¿Es útil o conveniente engañar al pueblo?» ${ }^{19}$. Bueno, ¿a ustedes qué les parece? De las 33 contribuciones aceptadas, 13 se pronunciaron en sentido afirmativo y 20 en sentido negativo. El jurado acordó repartir el premio entre los dos mejores ensayos representativos de una y otra postura: el afirmativo de Frederic de Castillon, profesor de matemáticas en la Academia Real de Gentilhombres, y el negativo de Rudolf Z. Becker, jurista y preceptor del barón de Dachenroede en Erfurt.

\footnotetext{
18 Véase A. Schopenhaver (1864, edición posterior), El arte de tener razón expuesto en 38 estratagemas, presentación y trad. de D. Garzón, Madrid, EDAF, 1996. Dialéctica erística o el arte de tener razón, expuesta en 38 estratagemas, introducción y trad. de L. F. Moreno Claros, Madrid, Trotta, 1997.

19 Condorcet, Castillón, Becker, ¿Es conveniente engañar al pueblo?, ed. de Javier de Lucas, Madrid, Centro de Estudios Constitucionales, 1991.
} 
Por ejemplo, según Castillón, conviene ocultar la verdad al pueblo para evitar desórdenes. Además, decirla sólo reportaría satisfacer la curiosidad o, a lo sumo, una especie de gratificación especulativa. «Con todo - advierte solícito-, seamos prudentes al disipar los errores; recordemos que no son un mal real hasta que se los conoce y que más vale un error útil que una verdad triste y estéril» (edic. c., p. 62). En cambio, según Becker, no sólo los ideales sino los impulsos naturales hacia la perfectibilidad humana en todos los órdenes traen como consecuencia la educación en la verdad y el destierro del prejuicio.

Así pues, he ahí la postura afirmativa de un matemático y la negativa de un jurista. ¿Cuál podría ser la de un filósofo? Al margen de la conocida opinión negativa del philosophe Condorcet, voy a aventurar una extrapolación que me sugiere precisamente nuestro homenajeado, José Luis Escohotado. Creo que si José Luis representara la posición filosófica, su alternativa sería de otro tipo: consistiría en hacer que la gente no se dejara engañar o, al menos, incluiría esta sana medida preventiva. También me parece que esta opción, a diferencia de los pronunciamientos más bien éticos o sociopolíticos en un sentido positivo o negativo, sería la que en principio adoptaría un teórico de la argumentación interesado en la lógica civil, es decir: interesado en poner a la gente en condiciones de asistir e intervenir lúcidamente en el discurso y en el conocimiento públicos.

Aunque también es cierto que, en este caso y en especial cuando concurren circunstancias sociales e ideológicas como las presentes, puede que ya no se trate sólo de teoría de la argumentación o de lógica civil, sino además de ciertas virtudes intelectuales y morales, como las que encarna José Luis: integridad, lucidez, clarividencia. Virtudes que, en fin, para redondear y cerrar a mi alegato en favor de la salud y de la calidad del discurso público, recomiendo reconocer, adoptar y seguir. 